\title{
El ejercicio de la autoridad en el aula, origen de desigualdades en el aprendizaje de la autonomía y de los hábitos democráticos ${ }^{1}$
}

The exercise of authority in the classroom, at the source of inequalities in the learning of autonomy and democratic behaviors

\author{
Sidonie Vacher \\ Universidad de Cádiz y \\ Universidad de Lyon 2, \\ Sidonie.Vacher@univ-lyon2.fr
}

\section{Resumen}

Nuestra investigación se centró en los vínculos que podrían establecerse entre la autoridad escolar, la autonomía de los individuos y las aspiraciones democráticas de las sociedades modernas, en el contexto francés. Nos enfocamos en dos concepciones contrarias de la autoridad que son: la autoridad con un fin educativo y la autoridad en una concepción autoritaria. En paralelo con un discurso político actual que aboga por la restauración de una autoridad autoritaria en las escuelas, un segundo objetivo político también se reafirmó en este momento; La Escuela debe formar el espíritu de los alumnos para examinar libremente y críticamente. El estudio cuestiona la naturaleza contradictoria de este doble mandato estatal y demuestra un trato desigual de los alumnos por parte del personal educativo en función de su nivel académico. Mostramos la injusticia cometida por la Escuela, en términos de empoderamiento y hábitos democráticos, al dirigir más la autoridad educativa hacia los alumnos con alto rendimiento escolar. Finalmente, damos claves para pensar la Escuela definitivamente de una manera distinta al modo de dominación-sumisión, profundizando en el pensamiento del filósofo John Dewey.

Palabras clave: Francia, Autoridad, Autonomía, Alumnos con bajo rendimiento académico, Alumnos con alto rendimiento académico, Hábitos democráticos, Desigualdad.

\footnotetext{
${ }^{1}$ Recibido: 27/02/2020 Evaluado: 09/03/2020 Aceptado: 19/03/2020
} 


\begin{abstract}
Our research focused on the links that could be established between school authority, the autonomy of individuals and the democratic aspirations of modern societies, in the French context. We focus on two contrary conceptions of authority that are: authority with an educational purpose and authority in an authoritarian conception. In parallel with a current political discourse that advocates the restoration of an authoritarian authority in schools, a second political objective was also reaffirmed at this time: The School must form the spirit of the students to think freely and critically. The study questions the contradictory nature of this double political injunction and demonstrates an unequal treatment of students by teachers based on their school level. We show the injustice committed by the School, in terms of empowerment and democratic habits, by directing more educational authority towards students with high school performance. Finally, we give keys to think the School in a different way to the mode of domination-submission, deepening the thinking of the philosopher John Dewey.
\end{abstract}

Keywords: France, Authority, Autonomy, Students with low school level, Students with high school level, Democratic habits, Inequality

\title{
Marco teórico del estudio
}

En el contexto de esta investigación, nos centramos en el tema de la autoridad en la escuela. La definición de autoridad depende de las diferentes concepciones que uno pueda tener de ella. Así, podemos mencionar tres, tal como las distinguió Robbes (2006), empezando por la autoridad educativa que requiere para existir el reconocimiento de los alumnos, su comprensión y su consentimiento. Esta autoridad se parece a una influencia educativa, porque no es una coacción ni una persuasión. Busca despertar en el otro una voluntad sin someterlo y es temporal porque, con su finalidad de emancipación de aquellos a quienes se dirige, esta autoridad educativa trabaja para su propia desaparición. También hay autoridad en su concepción autoritaria, que impone sin justificación, sin explicación, sin buscar la comprensión. Se trata en esta concepción, por el titular de una función estatutaria, de ejercer dominio sobre el otro en forma de sumisión, con el objetivo de su obediencia incondicional (Ibid.). El poder quiere ser indiscutible, la voluntad se impone unilateralmente. Esta es la autoridad a la que se refieren los varios políticos que piden la restauración de la autoridad en las escuelas. François Fillon, Ministro de Educación bajo la presidencia de Jacques Chirac, dijo en Le Monde de l'éducation que "desde el momento en que el profesor debe justificarse, su autoridad ya está cuestionada" ( «à partir du moment où le professeur doit de se justifier, son autorité est déjà entamée », Robbes, 2004). Nicolas Sarkozy también aboga por el regreso a la disciplina en la escuela, debido a una autoridad "sacudida" (Sarkozy, 2007, mi traducción). De manera similar, François Hollande, en el marco de la Gran Movilización de la Escuela por los Valores de la República, llama al personal educativo a informar al director 
de la escuela y nunca dejar sin consecuencia "cualquier comportamiento que ponga en peligro los valores de la República o la autoridad del docente" con la idea de que "es reforzando la autoridad del docente que compartiremos los valores republicanos" (Hollande, 2015, mi traducción). El actual ministro de la Educación Nacional, Jean-Michel Blanquer, también habla en su libro sobre la restauración de la autoridad para "restaurar la dignidad de la figura del maestro" («restaurer la dignité de la figure du maître», Blanquer, 2016). Finalmente, la tercera concepción que podemos mencionar es la autoridad evacuada, que surgió en la década de 1970 como una reacción a la autoridad autoritaria y se manifestó por la negativa a intervenir de los adultos, dudando de su legitimidad para ocupar una postura de autoridad. La evacuación de la autoridad por parte de los adultos, y la negación o el desafío de la autoridad por parte de algunos jóvenes en la actualidad (Rubi, 2010) vinculados a la falta de reconocimiento por parte de los alumnos de la asimetría educativa (Riondet, 2010) lleva a lo que muchos autores llaman la "crisis de la autoridad". Debido a ella, la cuestión de la autoridad en la escuela ha vuelto central. Como lo hemos dicho, numerosos políticos se posicionan a favor de una restauración de la autoridad autoritaria, y paralelamente a este comando político, una segunda orden estatal actual apunta a la introducción en los centros educativos de una formación al pensamiento crítico. El espíritu crítico, como un espíritu que rechaza cualquier argumento de autoridad y admite solo por verdadero lo que voluntariamente consiente después de un examen racional (Tozzi, 2002), se ha convertido en el enfoque intelectual de cualquier enseñanza (Éduscol, 2016) porque contribuye precisamente a uno de los principales objetivos de la institución escolar: hacer que los alumnos sean autónomos (Ibid.). Si se busca tanto esta autonomía de los alumnos es también porque es una condición sine qua non para la participación de los individuos en los procesos democráticos. Castoriadis hace del proyecto de autonomía una condición indispensable para la construcción de una "democracia radical", basada en "la ausencia de cualquier norma o ley extra-social que se imponga a la sociedad" (Castoriadis, 1986, p.479, mi traducción). En este sentido, una sociedad democrática es una sociedad autónoma, es decir, capaz de cuestionar constantemente la validez de las normas/leyes/instituciones y de transformarlas. Es porque los ciudadanos son autónomos que tienen la capacidad de cuestionar constantemente la ley y sus fundamentos, y es porque la cuestión de la norma siempre está abierta que una sociedad es justa (Castoriadis, 1979). Gutmann sostiene que el desarrollo de la autonomía ciudadana permite asegurarnos que las personas puedan participar activamente en los procesos democráticos (Gutmann, 1987). Las aspiraciones democráticas de nuestras sociedades modernas exigen que la Escuela participe en la educación ciudadana. Ahora bien, un elemento contradictorio nos interpela: ¿podemos participar en el empoderamiento de los alumnos y en el surgimiento en ellos de habilidades y valores democráticos mientras tenemos prácticas autoritarias hacia ellos? Este informe se centrará en cuestionar esta aparente contradicción. De manera similar, el presente estudio examinará si las concepciones autoritarias y educativas de la autoridad son igualmente aplicables a los alumnos, independientemente de su nivel académico. ¿Ofrece la Escuela las mismas oportunidades de emancipación y de participación futura en la vida democrática a sus alumnos? Primero presentaremos más precisamente los objetivos que subyacen a nuestro trabajo de investigación. Luego, expondremos la metodología elegida para llevar a cabo nuestro estudio, así como nuestras conclusiones. Por último, propondremos recomendaciones para la renovación del ejercicio de la autoridad en las escuelas. Nuestro estudio se inscribe, por lo tanto, en una perspectiva de mejora de la oferta educativa y de innovación docente. Debe 
tenerse en cuenta que este trabajo se centra exclusivamente en la escolarización en Francia y no pretende generalizar sus hallazgos más allá.

\section{Especificación de objetivos}

Como se explica anteriormente, la problemática central de este informe es la siguiente: ¿Ofrece la Escuela las mismas oportunidades de emancipación y participación futura en la vida democrática a sus alumnos? De hecho, el desafío detrás de este trabajo de memoria es verificar si la institución escolar está creando o no desigualdades en relación con el acceso a la autonomía y con la educación ciudadana.

Varios objetivos de trabajo se derivan de esta interrogación central.

1) Demostrar que la autoridad autoritaria se dirige más a los alumnos con rendimiento académico bajo y que la autoridad educativa está más destinada a los alumnos con rendimiento alto.

2) Demostrar que la Escuela crea graves desigualdades en términos de autonomía y de formación ciudadana debido a una socialización escolar democrática que no está dirigida de manera igualitaria.

3) Desarrollar nuestra propuesta de innovación docente para salir de la aporía, basándonos en la filosofía de John Dewey.

\section{Metodología}

En esta parte se presentará en dos etapas nuestra metodología de encuesta: primero el cuestionario en línea, luego las entrevistas con los expertos.

\section{Cuestionario en línea}

El cuestionario en línea se realizó en el marco de una encuesta universitaria en los meses de enero de 2018 a abril de 2018 (Université Lumière Lyon 2, Francia). Nuestro estudio anterior, que se prolonga en el año 2019 con la Universidad de Cádiz, se interesó en la población del alumnado francés de secundaria (aproximadamente 11-17 años). Los datos así recopilados provienen de adolescentes franceses que asisten a escuelas en el territorio. En este estudio se analizaron las respuestas de 65 estudiantes de educación secundaria.

Nuestro cuestionario (anónimo) tiene como objetivo conocer la percepción del alumnado de educación secundaria sobre el tema de la autoridad en la escuela y del pensamiento crítico. Se realizó utilizando la plataforma Lime Survey, y se difundió principalmente a través de las redes sociales. Para el análisis estadístico de los datos, nos basamos principalmente en Excel y Spad. Este cuestionario contiene 24 preguntas, y se divide en 3 partes: Una primera parte se interesa principalmente en recopilar datos sociodemográficos sobre el encuestado. La segunda fase se focaliza en la percepción de los alumnos en cuanto a la autoridad en la escuela. Buscamos en esta sección determinar a qué concepción se refiere el alumno cuando piensa en la autoridad en su trayectoria escolar. Por fin, la tercera parte cuestiona el pensamiento crítico de los alumnos en la escuela. 


\section{Consulta de expertos}

Para continuar esta investigación sobre la autoridad y el espíritu crítico en el marco de una investigación con la Universidad de Cádiz (UCA), decidimos consultar a expertos para compartir nuestras interrogaciones/reflexiones y recopilar sus opiniones. El presente estudio se extendió durante los meses de febrero de 2019 a mayo de 2019. Tres expertos participaron en este trabajo de investigación. En primer lugar, Agnès Grimault-Leprince, profesora universitaria de sociología en la ESPE de Bretaña (Francia), nos dio algunas respuestas por correo el 06/05/2019. Luego hablamos el 08/05/2019 durante una entrevista física, de tipo semiestructurado, con Juan Carlos Mougán Rivero, profesor de filosofía moral y política en la UCA, a cargo de un curso llamado "Teoría y práctica de la democracia" y especialista en filosofía de John Dewey. La segunda entrevista (semiestructurada) se realizó a través de la aplicación de Skype el 11/05/2019 con Bruno Robbes, profesor en la Universidad de CergyPontoise (Francia) y especialista en temas de autoridad. El objetivo era recopilar respuestas a las preguntas que nos preocupaban y obtener una opinión externa y experta sobre las conclusiones a las que llegamos. Sus aportes nos ayudaron a concretar nuestra propuesta final.

\section{Exposición de resultados}

El análisis de las respuestas del cuestionario demuestra la existencia de una diferencia en la percepción que los alumnos tienen de la autoridad, percepción que tiende a variar según el nivel académico de estos últimos. Mientras que la mitad de los alumnos con bajo rendimiento académico encuestados decidieron definir la autoridad como el "Poder para decidir u ordenar, para imponer sus deseos a los demás", el $83 \%$ de los alumnos con alto nivel escolar eligieron las otras definiciones en las que encontramos los términos "desarrollo", "poder legítimo" o "capacidad para hacer crecer". Por lo tanto, a diferencia de los alumnos con alto rendimiento escolar, los alumnos con un bajo nivel académico son numerosos en asociar la autoridad con la mera imposición de una voluntad externa sobre ellos. Entonces podemos considerar seriamente la existencia de un tratamiento diferenciado por parte de los docentes de los alumnos según su nivel escolar. La autoridad en su concepción educativa, en el sentido de que apunta al crecimiento de los individuos, y presupone el reconocimiento de estos últimos, parece dirigirse principalmente a los alumnos con alto rendimiento escolar, o al menos ser percibido como tal solo por una mayoría de estos últimos. La percepción de la autoridad por parte de los alumnos con un bajo nivel escolar se parece más a una autoridad autoritaria que impone y exige una obediencia incondicional.

Este reconocimiento desigual de la autoridad por parte de los alumnos se ve reforzado por el análisis de las preguntas que se refieren a la percepción de los encuestados en cuanto a las sanciones aplicadas en la escuela. Hay una clara diferencia en la percepción de la legitimidad de las sanciones: el 70\% de los alumnos con bajo rendimiento académico que participaron en nuestra encuesta consideraron que las sanciones impuestas en la escuela fueron injustas, en comparación con solo el $17 \%$ de los alumnos con un alto nivel escolar. De la misma manera, el sentimiento de los alumnos sancionados varía nuevamente según el nivel académico de estos últimos: ningún alumno con bajo nivel académico afirmó entender la 
sanción, mientras que el 33\% de los alumnos con nivel alto dijeron haberla entendido. Los alumnos con bajo rendimiento académico se sienten más humillados e indignados que sus compañeros con alto rendimiento después de un castigo. Una vez más, se está considerando una desigualdad en el tratamiento de estos alumnos por parte del personal educativo. Estos resultados apoyan y confirman el trabajo de Grimault-Leprince: los estudiantes con bajo rendimiento académico parecen estar afectados por una "represión pedagógica" que probablemente aumentará su sentimiento de injusticia y su incomprensión con respecto a las sanciones otorgadas (Grimault-Leprince, 2011). Estos sentimientos provocados por un acto de autoridad (como el castigo) están en total contradicción con lo que se ha definido como la autoridad educativa, porque esta última siempre debe ser entendida por el alumno, solo fuese para existir. Una vez más, la autoridad educativa parece ser más reservada a los alumnos académicamente fuertes que a los con dificultades escolares. Es necesario matizar nuestro punto, ya que también se encuentran los sentimientos de revuelta (23\%) y de humillación $(10 \%)$, aunque en una proporción menor, entre los mejores estudiantes.

Esta autoridad educativa también debe, como se ha visto anteriormente, ser discutible/impugnada para ser legítima. Sin embargo, esta oportunidad de impugnar, de expresar su opinión y sus sentimientos no es una posibilidad que se da a los alumnos por igual. Al cuestionar los encuestados en torno a su capacidad para impugnar si están en desacuerdo con una regla, una sanción o el discurso de un docente, el $70 \%$ de los alumnos con bajo rendimiento académico estimaron que no tenían la oportunidad para hacerlo porque los profesores no aceptan ninguno de sus comentarios, en contra del $40 \%$ de los alumnos con alto nivel académico. Lo que surge de nuestro tratamiento de datos es que la capacidad de impugnar no es la misma dependiendo del nivel académico del estudiante. Los docentes tienen más en cuenta los comentarios de los alumnos con alto rendimiento escolar (el 37\% de estos últimos pensaron que podían impugnar porque los profesores toman en cuenta sus observaciones, en comparación con el 10\% de los alumnos con bajo rendimiento académico).

Asimismo, solo el $10 \%$ de los alumnos con alto rendimiento escolar estimaron que tenían relaciones bastante malas con sus profesores porque no les permiten decir lo que piensan, contra el $40 \%$ de los alumnos con dificultades escolares. De manera similar, el $47 \%$ de los alumnos académicamente fuertes estimaron que tenían relaciones bastante buenas con sus docentes porque les escuchan, mientras que ningún alumno con bajo nivel académico ha elegido esta propuesta. Estas diferencias de percepción entre los encuestados una vez más ponen de relieve el trato desigual de los estudiantes por parte de los profesores debido a su nivel académico. Un buen alumno puede expresar más sus sentimientos e impugnar lo que hace autoridad en la escuela (ya sean las reglas, las sanciones, el discurso de un docente) que un alumno con dificultades escolares.

Lo que surge del análisis de los datos es que la autoridad en su concepción autoritaria está más dirigida a los alumnos con dificultades escolares, que no tienen la misma libertad de impugnación y expresión que sus compañeros con mejores resultados académicos. Por lo tanto, estos alumnos en dificultad deben más a menudo obedecer sin comprender, someterse sin negociar, lo que es típico de una autoridad autoritaria que impone sin discusión, sin explicación, sin consentimiento. Este hallazgo proporciona una mejor comprensión de las respuestas de los encuestados a la pregunta que cuestiona los alumnos sobre la actitud que creen que es más apreciada por sus docentes en el aula. El 27\% de los alumnos con alto 
rendimiento académico mencionaron la actitud "Participar para reaccionar ante los discursos de un docente/compañero, mostrar su desacuerdo, dar su opinión", mientras que ningún alumno con bajo rendimiento mencionó esta actitud. Esto refleja las expectativas diferenciadas de los profesores según el nivel académico del estudiante.

Una de las consecuencias que subyace a esta desigualdad de tratamiento es la formación desigual al pensamiento crítico de los alumnos. De hecho, tener un pensamiento crítico es el fruto de un aprendizaje, de una educación que hace que la mente examine libremente, que no conozca ningún argumento de autoridad, que no acepte nada sin cuestionar su valor. Sin embargo, parece que no todos los alumnos son educados de la misma manera al pensamiento crítico, porque los docentes no permiten que todos impugnen, discutan y expresen su opinión. Mientras que los estudiantes con mayor talento académico hacen más uso de su espíritu crítico en el aula, los con bajo nivel escolar no son permitidos hacer el mismo uso de su pensamiento o expresión crítica. La tercera parte de nuestro cuestionario interroga la capacidad de la escuela para permitir que los alumnos desarrollen y expresen su propia opinión. Lo que surge del análisis de las respuestas es, una vez más, una diferencia significativa entre la percepción de los niños con dificultades escolares y los de alto rendimiento académico: el $70 \%$ de los primeros creen que la Escuela les enseña solo de una determinada manera de ver las cosas y les ajusta a esta forma de pensar, frente al $17 \%$ de los alumnos con alto rendimiento académico. Por lo tanto, las expectativas de los profesores y el espacio de libertad que dejan a sus alumnos parecen depender de su nivel académico. El hecho de que ninguno de los encuestados con dificultades escolares piense que la Escuela les permite desarrollar su propio pensamiento y expresarlo (contra el 37\% de los alumnos con alto nivel escolar) es sintomático de una diferencia real en el tratamiento de esta categoría de alumnos por parte de los profesores.

Nuestro estudio demuestra a este respecto la persistencia de una tradición represiva y autoritaria de la Escuela, y esta principalmente destinada a los alumnos con dificultades académicas. Por lo tanto, las condiciones de aprendizaje de estos últimos difieren de las de sus compañeros con mejores resultados escolares, y su espacio de libertad y expresión parece reducido. Pero este tratamiento diferenciado también tiene un impacto en el contenido que se va a transmitir y en los objetivos de la educación nacional. Al no solicitar el pensamiento y la expresión crítica por igual entre sus alumnos, la Escuela no cumple su propósito, que es hacer del pensamiento crítico el enfoque intelectual de cualquier enseñanza para permitir la emancipación de todos los jóvenes franceses (cf. Eduscol). El acceso a la autonomía, como uno de las grandes finalidades que persigue la Escuela, no puede lograrse por igual por todos los individuos si no se les permite tener los mismos comportamientos emancipadores.

Pero esta desigualdad de acceso a la autonomía, además de constituir una gran injusticia creada por la Escuela, lleva a otra: la desigual posibilidad de participación en la vida democrática de la sociedad. De hecho, como se explica en el marco teórico, la autonomía de los individuos es una condición sine qua non para su participación activa en los procesos democráticos (Gutmann, 1987). No puede haber procesos democráticos y deliberativos sin individuos capaces de expresar sus opiniones, de contrarrestar los de otros, de no someterse a formas de autoridad que consideran ilegítimas, de respetar la palabra de los demás, de participar activamente en la construcción de las normas que deberán respetar, etc. Lo que nuestra investigación revela es que el modo de socialización democrático (Tozzi, 2005), es 
decir, el proceso a través del cual la Escuela favorece el surgimiento de una personalidad democrática en sus alumnos, no se dirige a todos por igual. Mientras los alumnos con alto rendimiento académico experimentan los valores y conductas democráticos durante su escolarización, los alumnos con dificultades académicas experimentan más prácticas y valores que contradicen el ideal de nuestras sociedades modernas. Es precisamente esta cuestión de experimentación de los valores y conductas, en el centro del pensamiento del filósofo John Dewey, que se desarrollará más profundamente en la parte "Propuesta" de este estudio.

\section{Discusión}

Según se ha desarrollado anteriormente, la presencia de una autoridad autoritaria en las escuelas socava la meta de emancipación de la institución escolar y es incompatible con las aspiraciones democráticas del mundo de hoy. El pensamiento del filósofo John Dewey nos da claves para salir de la aporía. De hecho, este filósofo ha abogado toda su vida a favor de una organización democrática de las sociedades modernas, que comenzaría en y por la Escuela. Pensamos sinceramente que sus contribuciones hacen posible considerar la educación de manera definitivamente diferente que en el modo de la dominación-sumisión. Es en apoyo del libro de Dewey Democracia y educación, del artículo de Joëlle Zask "L’élève et le citoyen, selon John Dewey" (2001) que resume la teoría de la educación del filósofo, y de las contribuciones de Carlos Mougán y de Bruno Robbes que construimos nuestra propuesta para mejorar el ejercicio de la autoridad y reducir las desigualdades en las escuelas.

\section{La teoría de la educación de John Dewey}

Primero es necesario aclarar el pensamiento de John Dewey, antes de precisar sus implicaciones en una propuesta de mejora del ejercicio de la autoridad en las escuelas. John Dewey (1859-1952) es un importante filósofo estadounidense de la corriente pragmática. Su filosofía se ha desarrollado en torno a la creencia de que la democracia participativa y deliberativa debe considerarse como un fin y un medio; Y que la Escuela es su condición de posibilidad. Es en este sentido que este autor nos ayuda a concienciar la vinculación entre educación y democracia.

Es apropiado explicar, ante todo, el sentido que le da John Dewey a la democracia. Él ve la democracia como "una experimentación continua e interminable", dependiente de la "formación de individualidades unificadas" (Zask, 2011, p.54, mi traducción). La primera expresión se refiere a la idea de que la democracia solo tiene sentido si es creativa, ya que no puede ser más que un estado activo de experimentación e invención. Como el mundo aún está en proceso, la política tiene como finalidad de inventar continuamente nuevas herramientas adaptadas a las condiciones de la época y de la sociedad. Por lo tanto, las reglas y normas en esta perspectiva no pueden ser fijas y los ciudadanos deben participar activamente en su renovación. La segunda expresión se refiere a la profunda convicción de Dewey de que la democracia no es solo un régimen político o una forma institucional externa, sino que es un modo de vida personal, en el que determinados hábitos y valores hacen parte integrante de la personalidad de los individuos. Aquí aparece el papel moral, social y político de la educación, que, a través de la formación de los ciudadanos, hace posible la existencia y la estabilidad de la democracia. Para él, la formación ciudadana, cuyo hace la finalidad de 
la institución escolar, no puede ser considerada como un tema especializado y distinto de los demás debido a la continuidad existente entre el alumno y el ciudadano (Zask, 2011). "Formar ciudadanos no consiste en desarrollar una facultad especial (el civismo)" sino en fomentar una "actitud general" o un "modo de vida" (Ibid., p.55, mi traducción). Por lo tanto, no puede haber dualismo entre el hombre y el ciudadano. En realidad, todas las disciplinas deben ponerse en una perspectiva cívica, ya que la democracia vive solo si los individuos han desarrollado ciertas actitudes enraizadas en su personalidad. Así, la Escuela debe estar diseñada en su totalidad de manera que permita el surgimiento de una personalidad democrática, ya que es "la forma embrionaria de la comunidad democrática" (Ibid., p.55, mi traducción).

Es en apoyo de estas reflexiones que Dewey desarrolló su teoría de la experiencia. Aplicado al campo de la educación, esto significa, como explica Joëlle Zask (2011), que el conocimiento nace de la participación activa del alumno a una experiencia educativa. El individuo va a reorientar su conducta (y, por lo tanto, aprender) de acuerdo con lo que experimentará, en la interacción con los fines que su entorno le ofrece. Entonces, debemos entender la educación como un proceso de adquisición de experiencias, experiencias que, según Dewey, deben ser orientadas hacia el mismo fin: la formación ciudadana/la organización democrática de las sociedades. El rechazo de todos los dualismos es uno de los puntos centrales de la filosofía de Dewey, ya que impiden la creación de "líneas de continuidad" (cf. entrevista Mougán). Según él, hablar de teoría y práctica es finalmente hablar de lo mismo, porque son dos elementos inseparables, tributarios entre sí. En este sentido, lo que el niño experimentará y los conocimientos que adquirirá proceden del mismo proceso, están interconectados. Lo mismo ocurre, como explica Carlos Mougán, con los medios y fines: "Hablar de fin y medio es como separar cosas que al principio no podemos separar porque no podemos conseguir un fin a través de los medios que estén desligados enteramente de este fin" (cf. entrevista Mougán). Por lo tanto, no tiene sentido pensar por separado los valores/grandes objetivos (fines) y las prácticas/técnicas (medios) que conducen a estos fines. Aprender para Dewey es, por consiguiente, siempre reorientar su conducta y adoptar nuevos "hábitos" (concepto central de Dewey) según la interacción vivida con el entorno, y esto solo es posible a través de la experiencia. Aquí entendemos el significado del "Learning by doing" y la convicción de Dewey de que aprender a través de la experiencia es la única manera de aprender.

Es precisamente porque Dewey siempre ha rechazado los dualismos que se opuso radicalmente a dos pedagogías: la pedagogía tradicional y la pedagogía "chicken soup". La primera se basa en la idea de que el cerebro del niño está vacío y maleable, y que el maestro solo tiene que imprimir conocimientos externos en su mente para que se asimile. Aquí hay una relación dogmática con el saber, ya que el conocimiento enseñado se considera desde el principio como válido e incontestable. Al niño se le dan fines fijos que no participó a construir y que, por consiguiente, no tienen nada que ver con él, ya que se imponen desde el exterior. Esta concepción de la educación no permite la renovación necesaria del presente, ya que no presta atención ni a las experiencias personales del individuo ni a las "nuevas necesidades que la dinámica de las relaciones sociales crea constantemente" (Zask, p.59, mi traducción). En cuanto a la segunda pedagogía, que también podemos llamar libertaria, se basa en la idea de que las disposiciones/potencialidades de los niños ya son significativas en sí mismas y que solo tenemos que permitir su libre desarrollo. Sin embargo, según Dewey, estas 
disposiciones de los jóvenes requieren estructuración y orientación, ya que solo se desarrollan en interacción con los fines que su entorno les propone. Es el papel del docente de diseñar escenarios educativos y orientarlos de manera que suceda este proceso de búsqueda cooperativa de soluciones que define a la democracia. Por lo tanto, juzga estas dos educaciones antidemocráticas, porque consideran solo uno de los dos factores que constituyen la experiencia (el hecho de hacer una experiencia y el objeto de la experiencia) (Zask, 2011). La primera excluye a los sujetos de la participación en la producción y organización del conocimiento, produciendo hábitos "perezosos" (cf. entrevista Mougán), de "infantilismo" y "servilismo" (Zask, 2001, p.61, mi traducción) y conduciendo al estatismo social. La segunda se niega a traer objetos para guiar la experiencia y estructurarla. Al negar el hecho de que las facultades de aprendizaje no son innatas, sino que se adquieren, esta educación hace que los alumnos sean más dependientes de su entorno social y familiar y contribuye al mantenimiento de las desigualdades.

Al revés del mantenimiento y estatismo social, es precisamente para Dewey esta posibilidad de renovación de las conductas individuales lo que hace democrático el método de la experiencia. Si estamos de acuerdo con Joëlle Zask para definir la democracia como "la institución colectivamente relevante de cambio" (2001, p.62, mi traducción), entonces debemos concebir la sociedad como continuamente nueva y la Escuela como un medio para asegurar la renovación de ella. Para John Dewey, el estatismo no es sinónimo de estabilidad, es la capacidad de una sociedad para renovarse lo que garantiza su perduración. Su filosofía podría describirse como "contextualista" en el sentido de que Dewey rechaza la idea de que existiría, independientemente del contexto, una verdad superior, establecida de una vez por todas y universal (cf. entrevista Mougán). La verdad no existe aisladamente: "tenemos que construirla nosotros entonces construimos con nuestro material, nuestro tiempo [...], pero no es fijo, no es permanente, no es estable es siempre modificable, siempre por definición incierto. Pero esto es la democracia. La democracia es la búsqueda permanente colectiva por la solución a los problemas." (cf. entrevista Mougán). Precisamente porque las soluciones se redefinen constantemente, la institución escolar debe tener en cuenta la novedad que cada uno trae consigo para que los ciudadanos puedan renovar sus objetivos comunes de manera permanente y así hacer que la democracia viva. En este sentido, la Escuela siempre debe apuntar el empoderamiento de los sujetos, su emancipación frente a todo lo que es dogma e imposición.

\section{Implicaciones del pensamiento de Dewey sobre el ejercicio de la autoridad en las escuelas}

La filosofía de Dewey proporciona claves para mejorar la práctica docente en un entorno educativo y hacerla coherente y compatible con las aspiraciones democráticas de nuestras sociedades. Presentaremos en esta sección nuestras recomendaciones para mejorar el ejercicio de la autoridad en las escuelas, confiando tanto en el pensamiento de Dewey (interpretado por J. Zask y C. Mougán) como en las contribuciones del especialista en cuestiones de autoridad Bruno Robbes. 
Entonces, hay esta idea central en Dewey que nos desarrollamos a partir del entorno que nos rodea, nuestro pensamiento está condicionado por él. En este sentido, Carlos Mougán argumenta que un entorno autoritario y jerárquico conduce a la formación de hábitos igualmente autoritarios y jerárquicos (cf. entrevista Mougán). La experiencia nos enseña que las órdenes y los saberes son dogmas que hay que ejecutar/asimilar sin buscar a confrontarlos, impugnarlos o cuestionarlos. Este modo de razonar de los individuos queda preso de estos esquemas de pensamiento autoritarios que son aquellos que han sido desarrollado en la interacción con su entorno (cf. entrevista Mougán). Ahora bien, es precisamente cuando las soluciones provienen del exterior y se imponen sin que nosotros hayamos contribuido a su construcción que nos enfrentamos a una situación antidemocrática. El autoritarismo representa precisamente la idea de que hay una verdad externa a mí y que mi rol respecto a ella es totalmente pasivo. Su existencia y persistencia, en la sociedad como en la escuela, se debe al hecho, según Bruno Robbes, de que permite circuitos cortos y respuestas inmediatas (cf. entrevista Robbes). La sumisión de los alumnos que supone conductas automáticas y obediencia incondicional se puede percibir como un método más acomodaticio y eficaz, frente a una autoridad que busca legitimidad y pide el consentimiento.

Sin embargo, en el primer método, ya no estamos en el registro educativo, sino en el de una relación de dominación-sumisión (cf. entrevista Robbes). Las verdades preestablecidas, las soluciones que se imponen sin negociación, etc. "son mecanismos de disminuir las capacidades de los alumnos para gestionar sus propios asuntos por tanto hacerlos menos autónomos, menos democráticos" (cf. entrevista Mougán). De hecho, si se presenta el docente como el único poseedor de la verdad, los alumnos no tienen ninguna razón para buscar otras soluciones por sí mismos y se vuelven dependientes de él. La emancipación de los sujetos no está permitida en un contexto autoritario que favorezca el desarrollo de hábitos de servilismo/perezosos y la dependencia a una autoridad externa. Si otorgamos a otros nuestro poder de decisión, nuestra responsabilidad y nuestro pensamiento crítico, lo hacemos al precio de nuestra libertad y empoderamiento (cf. entrevista Robbes).

Por el contrario, un entorno rico, abierto al diálogo y sensible a otras perspectivas lleva los individuos a adquirir hábitos tales como la flexibilidad, la sensibilidad, la cooperación, la apertura de mente, etc. Puesto que las cosas siempre están transformándose, y que la democracia es precisamente la forma colectiva para responder continuamente a los nuevos problemas, una escuela democrática debe por lo tanto garantizar que toda la comunidad educativa se sienta partícipe de estas soluciones cooperativas (cf. entrevista Mougán). Los hábitos democráticos serían, en palabras de C. Mougán, aquellos que "nos ayudan en afrontar los cambios y las transformaciones, que nos permiten dar una repuesta más inteligente, más abierta a las nuevas cosas que van sucediendo" (cf. entrevista Mougán). Por otro lado, ciertos hábitos nos encierran y nos bloquean para adaptarnos a nuevas circunstancias, como los hábitos desarrollados en el contexto de un entorno autoritario y dogmático.

La paradoja de la Escuela es cuando quiere desarrollar comportamientos democráticos en sus alumnos (pensamiento crítico, capacidad para opinar y escuchar al otro, para considerar siempre otras perspectivas, para cuestionar los hechos, debatir colectivamente, etc.), pero lo hace pensando los medios de manera desconectada con los fines. En realidad, por culpa de este tipo de razonamiento, la institución escolar está socavando sus propias lecciones de democracia. Cualquier enseñanza practicada en un contexto autoritario conlleva la enseñanza 
implícita del autoritarismo, porque la experiencia del autoritarismo conduce a la internalización de ciertos valores y hábitos que le son propios. Dado que "la experiencia educativa es una experiencia de transformación de los hábitos para generar los hábitos [...] que se caracterizan por ser más flexibles, más abiertos", y que los docentes pueden ser designados como "los grandes instrumentos de esta transformación educativa", entonces no pueden tener otros hábitos que los que deben despertar en sus alumnos (cf. entrevista Mougán). Lo que revela esta investigación es que existe una tensión entre una serie de hábitos democráticos que la institución escolar desea desarrollar en sus alumnos y los hábitos a veces fijos y cerrados de los docentes (Ibid.). Como Bruno Robbes nos recuerda con razón, "el joven necesita adultos con quienes pueda identificarse para crecer" (mi traducción, cf. entrevista Robbes). Dado que existe esta noción de identificación que entra en juego en la construcción del niño, las nociones de ejemplaridad, credibilidad y coherencia deben estar en el centro de las prácticas de enseñanza (cf. entrevista Robbes).

Parece justo vincular la pedagogía tradicional descrita por Dewey con la concepción autoritaria de la autoridad, y la pedagogía libertaria con la autoridad evacuada, ya que tienen valores y conducen a comportamientos similares. De hecho, la autoridad autoritaria, al igual que la pedagogía tradicional, consiste en una imposición externa que no tiene en cuenta el sujeto y sus experiencias. La autoridad evacuada, como la pedagogía libertaria, se basa de hecho en una definición errónea de la libertad que consiste en la ausencia de restricciones. A este respecto, Robbes nos explica que, en realidad, la ausencia total de autoridad no existe, que la autoridad "no puede no ejercerse" (mi traducción, cf. entrevista Robbes). Por eso, llama también a la autoridad evacuada "autoridad transferida", en el sentido de que si no es el maestro quien la ejerce, entonces otra cosa tendrá autoridad (cf. entrevista Robbes). Por lo tanto, parece que Dewey estaría a favor de una autoridad educativa, que se aparta tanto del dogma como del laissez-faire. Según él, el trabajo del docente es crear escenarios educativos que promuevan el desarrollo de habilidades individuales. Es necesario guiar la experiencia y dar objetos canalizadores, sin por ello construir el conocimiento en el lugar del alumno. De hecho, hemos visto que si no es el sujeto el que experimenta las cosas, él no las aprende.

Más concretamente relacionado con el tema central de esta investigación, la filosofía de Dewey proporciona claves para pensar el ejercicio de la autoridad en un entorno educativo. Como todos los campos de la educación deben abrirse a la democracia y sus valores, la autoridad también debe ser democrática. Una autoridad democrática es una autoridad que autoriza a los alumnos a cooperar colectivamente para definir las normas y reglas a las que acuerdan cumplir. También es una autoridad que no es fija, sino contextual, es decir, que está abierta a cambios, transformaciones, ajustes. Ya que en un mundo de incertidumbre no hay otras posibilidades para acercarse a la verdad que buscarla de manera colectiva y continua (cf. entrevista Mougán), los alumnos tienen su palabra que decir. Desde luego, hay un marco mínimo, el "no negociable", que se impone tanto a los alumnos como a los profesores (cf. entrevista Robbes). Asimismo, el límite a la organización democrática de una escuela es que el adulto sigue siendo el garante, porque existe una asimetría generacional y, debido a su lugar como niño, no se le puede dar al alumno responsabilidades más allá de sus propias capacidades (cf. entrevista Robbes). Sin embargo, la democracia no es un estado natural, se construye y se aprende. Ser ciudadano el día de su mayoría no tiene sentido si no ha aprendido de antemano lo que constituye su ciudadanía (cf. entrevista Robbes). Por lo tanto, teniendo en cuenta este límite, el debate puede perfectamente ser la herramienta para resolver los 
problemas. Al funcionar así, cada miembro de la comunidad educativa es conducido a desarrollar sus habilidades ciudadanas.

De hecho, es a través del intercambio de opiniones por el bien común que se obtienen soluciones justas para todos. Por consiguiente, el proceso de deliberación debe impregnar todas las esferas escolares, e incluso la personalidad de los individuos. En este proceso, el acuerdo se logra no mediante la simple agregación de preferencias individuales, sino mediante la transformación de las convicciones a través del diálogo. Es precisamente la expresión de diferencias y desacuerdos lo que enriquece el proceso deliberativo y conduce a una síntesis/consenso que integra a todas las partes. No todas las opiniones son equivalentemente éticas, y el análisis racional e imparcial de estas últimas permite jerarquizarlas. Es un modelo político mucho más inclusivo que los anteriores y digno de nuestras capacidades humanas. A través de la experiencia del debate, los alumnos reconocen la importancia de las reglas en los que permiten la comunicación y la convivencia. Ellos son llevados a participar todos sin atención a sus características socio-académicas. Es precisamente este modelo político el que debería servir como forma de resolver los conflictos en las escuelas. No puede ser de otra manera en una sociedad que quiere ser democrática, e incluso si la tarea puede ser compleja, este es el papel de los docentes.

\section{Recomendaciones para salir de la aporía}

Encontramos en los escritos de Dewey, más que técnicas precisas que no tuvieran sentido aplicadas como tal en contextos distintos, pautas generales para la práctica docente. Si los interpretamos en el marco de nuestro tema y que incluimos las contribuciones de los expertos entrevistados, podemos, a modo de resumen, enumerar algunas claves para el ejercicio de la autoridad en las escuelas:

- Experimentar es reorientar su conducta en la interacción con el entorno. Y reorientar su conducta es aprender.

- Una enseñanza practicada en un contexto autoritario conduce al aprendizaje de valores y comportamientos específicos del autoritarismo.

- Los medios y fines no pueden ser desconectados. La experiencia que debe llevarnos a un fin y el fin de esta experiencia debe ser del mismo carácter y llevar los mismos valores, no son disociables.

- La autoridad no puede ser autoritaria ni evacuada. Es necesariamente educativa porque es la única concepción que tiene en cuenta los dos factores que constituyen la experiencia educativa, a saber, el hecho de hacer una experiencia (que la concepción autoritaria ignora al buscar la asimilación y la sumisión a través de la imposición) y la finalidad de la experiencia (que la concepción evacuada ignora al negar dar objetos canalizadores).

- El objetivo de una sanción educativa es permitir que el alumno asuma la responsabilidad de sus acciones. Sin embargo, no podemos empoderar al otro mediante sanciones repetitivas, similares y humillantes contra él. Castigar apunta a no tener que castigar más (cf. entrevista Robbes). 
- Ser capaz de cuestionar las intenciones de los alumnos y descifrar el porqué de su comportamiento es la condición para proporcionar una respuesta adecuada de naturaleza educativa (cf. entrevista Robbes).

- La continuidad y la coherencia son indispensables entre los comportamientos/valores del docente y aquellos que desea despertar en el alumno.

- La única forma justa de resolver conflictos es una basada en el modelo de deliberación, es decir, una que busca el consenso a través de la cooperación y el intercambio de argumentos sobre el bien común.

- El mundo siempre está transformándose, por consiguiente, las reglas y los principios no pueden ser fijados en el tiempo y deben ser reexaminados constantemente en función del contexto.

- Dado que la democracia es la renovación permanente y la búsqueda colectiva de soluciones, conviene que los individuos desarrollen hábitos que les permitan adaptarse a nuevas circunstancias y no bloquearlos.

- Finalmente, la autoridad docente consiste en diseñar escenarios educativos que permitan a los alumnos construir colectivamente los conocimientos y normas. Otorga a los alumnos un papel importante y los prepara activamente para su futura participación en la vida democrática.

\section{Conclusión}

El presente trabajo de investigación se centró sobre la autoridad en las escuelas francesas, su percepción por los alumnos franceses y su puesta en perspectiva con el empoderamiento y el desarrollo de una personalidad democrática. Nuestros resultados muestran un vínculo significativo entre la autoridad educativa y los alumnos con alto rendimiento académico, ya que estos últimos están más autorizados por sus docentes para argumentar, cuestionar y dar sus opiniones. Por otro lado, la autoridad en su concepción autoritaria está más dirigida a los alumnos con bajo rendimiento académico, que son menos autorizados a impugnar, expresar sus sentimientos y desarrollar una opinión propia en la escuela. Deben obedecer más sin comprender, sometiéndose sin negociar lo que es sintomático de las relaciones de dominación-sumisión. Las dos autoridades no son iguales en el plano educativo, porque una favorece el propósito de la Escuela, que es hacer que los alumnos sean autónomos al formar su espíritu para que examine críticamente, mientras que la otra lo perjudica peligrosamente. Debido a que no todos los alumnos pueden tener los mismos comportamientos emancipatorios, no todos desarrollan hábitos y valores democráticos. De hecho, ciertas habilidades despertadas en el contexto de la formación al pensamiento crítico, como expresarse con claridad - no someterse a formas autoritarias de autoridad - contribuir a la elaboración de las normas bajo las cuales uno acepta vivir, son requisitos previos fundamentales para la participación de los individuos en los procesos democráticos de nuestras sociedades modernas. La filosofía de John Dewey completa nuestro hallazgo. Este último insiste de manera radical en el hecho de que la democracia de hoy y mañana solo puede ser efectiva a través de la democracia en la escuela. Un individuo es verdaderamente 
un ciudadano solo si se le permite recibir la educación que le hará capaz de participar en la organización de la sociedad de la que es miembro. Nos convertimos en ciudadano a través de la trayectoria escolar, no solo es un estatus, sino el producto de una educación. El papel de los docentes aquí es primordial, ya que deben suscitar en sus alumnos hábitos democráticos que se vuelven a hacer parte de su personalidad.

Es por esto que también hemos desarrollado recomendaciones para que los profesores mejoren el ejercicio de la autoridad. El objetivo de estas recomendaciones es orientar la práctica docente para que la autoridad ya no sea un obstáculo para el empoderamiento y el surgimiento de una personalidad democrática, sino que autorice y promueva estos procesos. Nuestras propuestas alimentan las reflexiones sobre la innovación en el entorno educativo. De hecho, la innovación es una noción que resuena fuertemente en la filosofía de John Dewey, ya que está estrechamente vinculada a los conceptos de cambio y reforma (Arenas Martija y Margalef García, 2006). "Se innova para generar cambios” (p.15), para renovar el existente. Nuestras pistas para mejorar responden a las diferentes características de la innovación educativa identificadas por Arenas Martija y Margalef García (2006): el proceso de innovación en primer lugar supone una "idea percibida como novedosa por alguien". Implica "un cambio que busca la mejora de una práctica educativa" y conlleva un aprendizaje por los actores involucrados. Finalmente, cualquier innovación educativa está influenciada por intereses económicos, sociales o ideológicos (p.16).

La autoridad educativa tal como la defendemos se puede definir, con respeto a las prácticas actuales de autoridad en las escuelas, como una idea innovadora que implica un cambio en nuestras representaciones tradicionales de las relaciones entre adultos y niños. Es a fin de mejorar la calidad del proceso educativo que este cambio nos parece necesario, porque una autoridad autoritaria, por los sentimientos que despierta y las desigualdades que genera, es perjudicial para el aprendizaje. Implementar un modo de regulación educativo y democrático en su clase requiere conocer las consecuencias que generan otras formas de autoridad y, por lo tanto, requiere un aprendizaje previo que nuestro artículo pretende favorecer. Del mismo modo, el ejercicio de esta nueva forma de autoridad implica compartir una cierta visión de la educación, ciertos valores e ideales sociales. Las contribuciones de Dewey constituyen un background ideológico en nuestra propuesta de innovación: nuestra concepción de lo que es la buena autoridad está influenciada por el ideal democrático moderno y el propósito reciente de la Escuela para la emancipación de sus alumnos.

\section{Referencias}

Arenas Martija, A. y Margalef García, L. (2006). ¿Qué entendemos por innovación educativa? A propósito del desarrollo cuticular. Perspectiva Educacional. Formación de Profesores, 47, 13-31.

Blanquer, J-M. (2016). L'Ecole de demain. Paris: Edition Odil Jacob.

Boileau, X. (2016). Dewey et Gutmann. L'éducation civique comme nécessité démocratique. Ithaque, 19, 3-24. 
Castoriadis, C. (1979). Le contenu du socialisme. 1018.

Castoriadis, C. (1986). Les carrefours du labyrinthe II. Le Seuil.

Dewey, J. (1916). Democracy and Education. New York: Macmillan.

Foray, P. (2009). Trois formes de l'autorité scolaire. Le Télémaque, 35(1), 73-86.

Grimault-Leprince, A. \& Merle, P. (2008). Les sanctions au collège: Les déterminants sociaux de la sanction et leur interprétation. Revue française de sociologie, 49(2), 231-267.

Grimault-Leprince, A. (2011). La gestion de la classe par les enseignants de collège. Formalisme versus pragmatisme. Carrefours de l'éducation, 31(1), 217-235.

Gutmann, A. (1987). Democratic Education. Princeton, New Jersey: Princeton University Press.

Houssaye, J. (1996). Autorité ou éducation? Paris, E.S.F.

Kojève, A. (2004). La notion d'autorité. Paris : Gallimard coll. « Bibliothèque des idées ».

Meirieu, P. (2005). Quelle autorité pour quelle éducation ? [En línea]. Article présenté aux Rencontres Internationales de Genève. Recuperado el 14 de mayo de 2019, de: https://cutt.ly/rtzoWcy

Merle, P. (2005). L'élève humilié. L'école : un espace de non-droit ? Paris : PUF, Éducation et formation.

Ministère de l'Education nationale, de l'Enseignement supérieur et de la Recherche. (2016). A l'école de l'esprit critique. [En línea]. Recuperado el 14 de mayo de 2019 , de: https://urlz.fr/axKV

Prairat, E. (1997). La sanction : petites méditations à l’usage des éducateurs. Paris : Éditions l'Harmattan.

Prairat, E. (2003). La sanction en éducation. Paris : Presses universitaires de France.

Prairat E., (2010). «L'autorité éducative au risque de la modernité », in Prairat E., L'autorité éducative : déclin, érosion ou métamorphose, Nancy : Presses Universitaires de Nancy, «Questions d'éducation et de formation » pp.39-52.

Riondet, X., (2010). "L'Eduction nouvelle et la question de l'autorité », in Prairat E., L'autorité éducative : déclin, érosion ou métamorphose, Nancy : Presses Universitaires de Nancy, «Questions d'éducation et de formation », pp. 87-106. 
Robbes, B. (2004). L'autorité après la circulaire « Fillon » : questions de sens et de faire. Cahiers pédagogiques.

Robbes, B. (2006). Les trois conceptions actuelles de l'autorité. Site du CRAP Cahiers pédagogiques. [En ligne]. (pp.1-20). [En línea]. Recuperado el 14 de mayo de 2019 https://cutt.ly/OtzomT7

Rubi, S., (2010). «Le sexe de l'autorité », in Prairat E., L'autorité éducative : déclin, érosion ou métamorphose, Nancy : Presses Universitaires de Nancy, «Questions d'éducation et de formation », pp. 67-86.

Sarkozy, N. (2007). L'intégralité de la "lettre aux éducateurs » de Nicolas Sarkozy. Le Monde. [En línea]. Recuperado el 14 de mayo de 2019, de: https://2doc.net/hmh5n

Tozzi, M. (2002). L'éveil de la pensée réflexive à l'école primaire. Hachette Education.

Tozzi, M. (2005). «L'autorité démocratique » : une provocation conceptuelle ? Les Cahiers du Cerfee, 21.

Zask, J. (2001). L'élève et le citoyen, d'après John Dewey. Le Télémaque, 20 (2), 53-64.

\section{Principales referencias videográficas}

Los deseos de François Hollande al mundo de la educación, de la educación superior y de la investigación el 21 de enero de 2015 - Video publicado por Le Figaro en Dailymotion el 21/01/2015. Recuperado el 14 de mayo de 2019, de: https://2doc.net/6ksex 